\title{
II terrapiattismo e i suoi sostenitori
}

\author{
Miriam Di Carlo
}

PUBBLICATO: 31 LUGLIO 2019

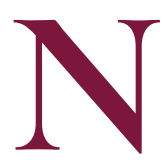
egli ultimi tempi, si sente spesso parlare di terrapiattismo (anche con la lettera maiuscola Terrapiattismo) e terrapiattista, due nuove parole con cui si indicano rispettivamente la corrente di pensiero secondo cui il pianeta Terra è piatto' e "chi, che crede che il pianeta Terra sia piatto" (definizione di Treccani Neologismi 2019). L'impiego di queste due nuove parole ha conosciuto un netto incremento negli ultimi tempi: basti pensare che l'is aprile zorgterrapiattismo aveva su Google (pagine in italiano) 4r.ooo occorrenze, mentre il I5 maggio 2019 (poco piu di un mese dopo) 97.600. Cosi come terrapiattista che da 29.800 occorrenze è passato a 50.800; più evidente la crescita con il plurale terrapiattisti che è passato da 267.000 a 488.000 occorrenze.

Sebbene questa corrente di pensiero trovi le sue origini in ambiente inglese e poi americano nella Flat Earth Society (da Flat 'piana' Earth 'Terra'), i cui adepti sono chiamati flat-earthers, in Italia si sono diffuse queste due parole create con elementi della lingua italiana. Infatti la parola terrapiattismo è formata da una base sintagmatica (Terra piatta) e dal suffisso -ismo, uno dei più produttivi in italiano, che può essere usato per formare parole che indicano concezioni politiche (ad es. sovranismo di Matilde Paoli e cfr. anche populismo di Giuseppe Patota), religiose, filosofiche, artistiche, scientifiche ecc. Il suffisso -ismo risulta particolarmente produttivo nella formazione di parole afferenti al campo filosofico (empirismo) e a quello delle scienze dell'uomo (strutturalismo), non solo per indicare le discipline ma anche "per riferirsi a determinati fenomeni scientifici, ma in misura molto più ridotta: magnetismo [...], vulcanismo" (Grossmann-Rainer 2004, p. 259). Il suffisso -ismo risulta appropriato per una parola come terrapiattismo che si riferisce a una corrente di pensiero che ha un'eco filosofica e/o scientifica. Vale la pena soffermarsi sulla base della parola: Terra piatta. In riferimento alla credenza e al movimento che la sostiene, questo sintagma, composto da sostantivo + aggettivo sta assumendo le caratteristiche di una polirematica ovvero di un'unità lessicale superiore (formata da più termini), caratterizzata dall'impossibilità di inserire materiale linguistico al suo interno (del tipo *Terra sostanziale piatta). Sempre più spesso ricorrono, sui giornali e non solo, sintagmi e frasi del tipo: $i$ sostenitori della Terra piatta, la corrente della Terra piatta. Nel caso di terrapiattismo ci troviamo di fronte dunque a una parola nuova interamente italiana formata attraverso meccanismi di composizione (Terra + piatta) e di derivazione (con il suffisso -ismo). A volte si possono trovare le grafie Terra piattismo e Terra-piattismo (cosi come Terra piattista e Terra-piattista) ma sono poco frequenti e quindi poco rilevanti. Inoltre, avendo come base un nome proprio (il pianeta Terra), il derivato spesso presenta la lettera maiuscola che in realtà non è necessaria perché il nome derivato è un nome comune: basti pensare a marxismo, calvinismo ecc. Un discorso simile si può fare per terrapiattista. Il suffisso -ista (di origine greca -istēs), nonostante oggi sia spesso usato in funzione aggettivale, nasce in realtà per formare sostantivi che derivano da nomi propri: il 'seguace di X' o anche 'chi segue e applica una convinzione e spesso anche con ammirazione, le preposizioni formulate da X'. In questo caso il terrapiattista è il seguace della corrente della Terra piatta, del terrapiattismo. Le due parole non sono registrate in nessuno dei dizionari contemporanei eccezion fatta per il repertorio Treccani, sez. Neologismi 2019. Si è inclini a pensare che di solito nasca prima la parola che indica il movimento (il questo caso terrapiattismo) e poi quella indicante la persona che si riconosce in tale movimento (terrapiattista). In questo caso particolare pero non possiamo esserne certi visto che, stando alle attestazioni rilevate sul web, la prima attestazione di terrapiattista risale al 20 Io mentre 
quella di terrapiattismo al 20I:

Il terrapiattista convinto che basti ragionare un attimo per vedere l'assurdità delle teorie astruse degli scienziati (commento di Gianni Comoretto sul blog ugobardi.blogspot.com, 6/6/2010).

Saccenteria al servizio del terrapiattismo, esibita da chi probabilmente era ancora uno sbarbatello quando Kiehl e Trenberth pubblicavano questo paper: è questa l'ultima provocazione con parvenza di scientificità (post sul blog climafluttuante.blogspot.com, 25/2/20II).

Bisogna considerare che il modello di riferimento per il terrapiattismo italiano è l'ambiente angloamericano in cui, come si è detto, il movimento viene indicato con il nome della società: Flat Earth Society. Eventualmente ci sono casi in cui tale corrente viene indicata solo con Flat Earth (a cui si ispira il sintagma italiano Terra piatta) mentre non esiste un *flatearthism. Invece, gli adepti della Flat Earth Society nonché i sostenitori della corrente di pensiero vengono dettiflat-earthers, parola che risulta molto frequente all'interno dei testi in lingua inglese. In italiano, la parola terrapiattista sembra essere un calco morfologico diflat-earther, caso simile a quello di skyscraper-grattacielo e dunque potremmo supporre, rifacendoci ai pochi dati a disposizione, che la parola terrapiattista sia stata formata prima di terrapiattismo e non viceversa.

Per quanto riguarda i testi in lingua italiana, le attestazioni di terrapiattismo e terrapiattista dal zorI (anno della sua comparsa) sono sporadiche fino al 2016-20I7, anni a partire dai quali Google Trends registra un costante crescendo di ricerche sul motore di ricerca:

Fritto misto terrapiattista (Mondi sommersi): ... serie coi buchi) quando Bush entrava nella Casa Bianca ... ( tweet di @BarackObamaIT del 23/r/20II).

Se il terrapiattismo fosse una religione ufficiale, potremmo dire che il suo capostipite e profeta sarebbe senza dubbio Cosma Indicopluestes. (post sul blog documentazione.info, 25/3/2013).

PS Ma oltre a questi pallosissimi video di youtube il terrapiattismo non ha prodotto altre prove? (commento sul forum old.luogocomune.net, 24/8/2014).

Non conoscevo questa affermazione a riguardo del sistema tolemaico. SI mostra quindi veramente all'avanguardia, nelle intenzioni. Poi sappiamo che invece e' [sic] stato assurto a simbolo di "retrogradi contro progressisti” e da li' [sic] gli son piovuti addosso i peggiori insulti, non ultimo quello completamente inventato di terrapiattismo ( commento sul blog enzopennetta.it, I/I2/2014).

Quando il terrapiattismo salterà il fosso e si assumerà le sue responsabilità? Vogliamo illuminare le menti e far comprendere a tutti in quale abissi [sic] di ignoranza ci hanno confinato. Compagni terrapiattisti, formiamo un movimento e candidiamoci alle prossime elezioni ( commento al blog di repubblica.it, disgrazia-lescienze.blogautore.espresso.repubblica.it, I3/II/2015).

La seguente tabella si riferisce alle occorrenze su Google (pagine in italiano) di terrapiattismo/i e terrapiattista/i/e divise per anni. Si consideri che nel caso di terrapiattista, il singolare può essere tanto aggettivo quanto sostantivo (con prevalenza di quest'ultimo), il plurale terrapiattisti viene usato quasi esclusivamente come sostantivo mentre il plurale terrapiattiste quasi esclusivamente come aggettivo (del tipo le teorie terrapiattiste): 


\begin{tabular}{|c|c|c|c|c|c|c|c|c|c|c|c|}
\hline GOOGLE (p.i.) & 2011 & 2012 & 2013 & 2014 & 2015 & 2016 & 2017 & 2018 & $7 / 5 / 2019$ & \multicolumn{2}{|c|}{$15 / 5 / 2019$} \\
\hline $\begin{array}{l}\text { terrapiattismo } \\
\text { terrapiattismi }\end{array}$ & $\begin{array}{c}152 \\
0\end{array}$ & $\begin{array}{c}198 \\
0\end{array}$ & $\begin{array}{c}107 \\
0\end{array}$ & $\begin{array}{c}146 \\
0\end{array}$ & $\begin{array}{c}142 \\
0\end{array}$ & $\begin{array}{c}323 \\
0\end{array}$ & $\begin{array}{c}958 \\
0\end{array}$ & $\begin{array}{c}3.910 \\
1\end{array}$ & $\begin{array}{c}3.220 \\
8\end{array}$ & $\begin{array}{l}7.500 \\
8\end{array}$ & $\begin{array}{l}(+4.280) \\
(+0)\end{array}$ \\
\hline $\begin{array}{l}\text { terrapiattista } \\
\text { terrapiattisti } \\
\text { terrapiattiste }\end{array}$ & $\begin{array}{c}10 \\
947 \\
0\end{array}$ & $\begin{array}{c}10 \\
750 \\
0\end{array}$ & $\begin{array}{c}96 \\
977 \\
0\end{array}$ & $\begin{array}{c}8 \\
1.200 \\
1\end{array}$ & $\begin{array}{c}172 \\
2.140 \\
0\end{array}$ & $\begin{array}{c}318 \\
8.570 \\
4\end{array}$ & $\begin{array}{c}551 \\
7.830 \\
19\end{array}$ & $\begin{array}{c}2.430 \\
17.300 \\
71\end{array}$ & $\begin{array}{c}2.100 \\
12.000 \\
105\end{array}$ & $\begin{array}{l}2.700 \\
69.700 \\
119\end{array}$ & $\begin{array}{l}(+600) \\
(+57.700) \\
(+14)\end{array}$ \\
\hline
\end{tabular}

I dati della tabella mostrano che l'incremento di occorrenze comincia nel 20I6. Anche il 2018 registra molte attestazioni ma l'anno decisamente più ricco di occorrenze si rivela essere il 2019. Si è deciso di inserire due colonne distinte per il zorg perché il I2 maggio 2019 si è tenuto a Palermo un incontro dei terrapiattisti italiani che ha fatto parlare di sé giornali, telegiornali, forum, blog. A conferma dei dati di Google, ci sono quelli di Twitter che rivelano una situazione del tutto analoga:

\begin{tabular}{|c|c|c|c|c|c|c|c|c|c|l|l|}
\hline $\begin{array}{c}\text { TWITTER } \\
\text { (lingua italiana) }\end{array}$ & $\mathbf{2 0 1 1}$ & $\mathbf{2 0 1 2}$ & $\mathbf{2 0 1 3}$ & $\mathbf{2 0 1 4}$ & $\mathbf{2 0 1 5}$ & $\mathbf{2 0 1 6}$ & $\mathbf{2 0 1 7}$ & $\mathbf{2 0 1 8}$ & $\mathbf{7 / 5 / 2 0 1 9}$ & $\mathbf{1 5 / 5 / 2 0 1 9}$ \\
\hline $\begin{array}{c}\text { terrapiattismo } \\
\text { terrapiattisti }\end{array}$ & 0 & 0 & 0 & 0 & 0 & 13 & 81 & 89 & 78 & 160 & $(+82)$ \\
\hline terrapiattista & 1 & 0 & 0 & 0 & 0 & 0 & 1 & 7 & 13 & 16 & $(+3)$ \\
terrapiattisti & 0 & 0 & 1 & 0 & 0 & 29 & 53 & 78 & 113 & 284 & $(+171)$ \\
terrapiattiste & 0 & 0 & 0 & 0 & 0 & 1 & 15 & 42 & 24 & 400 & $(+258)$ \\
$(+5)$
\end{tabular}

Le attestazioni di terrapiattismo e terrapiattista fino al 2017 provengono da blog sia individuali (ovvero tenuti da un solo opinionista) sia collettivi, da commenti e da discussioni sui forum, da pareri espressi sui social (in particolare Twitter). Lidea che la Terra possa essere nuovamente considerata piatta è un argomento che, a quanto pare fa dibattere e discutere. Nel 2017 cominciano le prime attestazioni sui giornali su cui però spesso, si preferisce mettere le virgolette:

Il soprannome che si è dato corrisponde a quello che in parecchi pensano di lui. Un folle, che ha già fatto parlare di sé, e non solo per l'adesione al "terrapiattismo" (Matteo Marini, "Voleró con il mio razzo per dimostrare che la Terra è piatta", articolo in repubblica.it, 22/II/2017).

Dopo il fallimento di una prima campagna di 'fundraising' su Kickstarter Hughes, convertitosi solo recentemente al "terrapiattismo", è tornato alla carica focalizzando la sua ricerca di sponsor all'interno della comunità che condivide il suo credo ([s.f.], "La Terra é piatta", e per dimostrarlo si lancia in volo con un razzo fai da te, articolo in rainews.it, 22/II/2017).

I terrapiattisti - cosi si chiamano i sostenitori della tesi per cui la Terra sia [sic] piatta - sono molto attivi sul web e sui social network e hanno addirittura un sito ufficiale (The Flat Earth Society), dove sono presentate le varie prove del terrapiattismo ([s.f.], Ma se gia gli antichi greci dicevano che la Terra era rotonda, perché queste celebritá si ostinano a dire che é piatta?, articolo in huffingtonpost.it, 16/12/20I7).

Oggi tutti i quotidiani nazionali usano nei loro articoli le parole terrapiattismo e terrapiattista grazie all'attenzione che sta ottenendo il movimento della Terra piatta. I termini sono comparsi anche sui libri già dal 20I5, pur registrando sporadiche occorrenze almeno fino al 20I8:

Scacciai dalla mente quel pensiero per accorgermi che Francesco aveva lo sguardo sognate e un po' folle di chi si prefigura il patto come una specie di gioioso happening, un grande metaforico abbraccio universale in cui chiunque, dagli amici terrapiattisti alla custode del palazzo, può andare e venire (Paola Maraone, Paola La Rosa, Straziami ma di tofu saziami, Milano, Rizzoli, 2015).

Già dalle prime occorrenze dei termini si colgono nuove sfumature di significato del termine terrapiattista che viene sempre più considerato un sinonimo di 'incolto', 'ingenuo', 'idiota' 'persona 
affetta da manie di persecuzione' e anche 'anticonformista'. Il perché di questo sviluppo semantico va ricercato nelle idee che vengono sostenute dal terrapiattismo. Anzitutto, come si accennava all'inizio, il movimento italiano del terrapiattismo si ricollega a quello inglese e americano della Flat Earth Society nato in Inghilterra negli anni '5o sulla scorta di teorie e movimenti preesistenti. Le teorie della Terra piatta trovano uno scoglio nelle foto scattate negli anni '6o dalla NASA durante le spedizioni sulla Luna che proponevano un pianeta Terra decisamente sferico. Da questo momento in poi i flat-earthers (come sono chiamati i terrapiattisti in inglese), attribuiranno alle autorità mondiali (il governo degli Stati Uniti, le entità da esso gestite come la NASA e poi le autorità di potere in generale) la responsabilità di voler nascondere al mondo intero una serie di verità, con lo scopo di tenere nell'ignoranza la popolazione mondiale per assoggettarla e manovrarla piu facilmente.

Le teorie della Flat Earth Society dunque non riguardano solo la questione della forma del pianeta Terra, ma coinvolgono altri interrogativi arrivando a sostenere l'idea di un grande complotto ordito dalle autorità mondiali, a scapito di tutta l'umanità. Il terrapiattismo italiano, sviluppatosi grazie alla proliferazione di informazioni su internet (soprattutto tramite una serie di video su YouTube), ha mutuato in toto le idee della Flat Earth Society, arrivando a considerare i governi nazionali, tra cui quello italiano, gli attori di un grande complotto internazionale, reso possibile grazie al controllo delle menti che verrebbe esercitato fin dall'infanzia tramite la scuola, i libri di studio, poi la tv, i giornali ecc. Considerando la storia della Flat Earth Society e del terrapiattismo italiano si comprende che le accezioni a cui abbiamo accennato (tranne quella di 'anticonformista'), nascono dal giudizio espresso sui terrapiattisti da chi non crede alle loro teorie.

Sono significative le parole con cui Stefano Bartezzaghi parla della corrente di pensiero e dei suoi sostenitori:

\begin{abstract}
persino dell'enunciato "La Terra è piatta" il quale, come sappiamo, negli ultimi anni ha avuto un numero impensabile di adesioni. Le teorie cosiddette "terrapiattiste" sono il grado estremo della potenzialità controfattuale dei social perché attaccano una delle banalità più esemplari, cioè "la Terra è rotonda". Esemplare perché viene appunto citata comunemente come verità fuori discussione, appena un gradino sotto "due più due fa quattro". Fino al recente passato si è ritenuto che a credere che la Terra non sia rotonda potessero essere solo figure umane di arretratezza pressoché inimmaginabile per deficit scolastico o cognitivo gravissimo [...] il caso del "terrapiattismo" ci mostra come l'adesione a un enunciato sia completamente indipendente dal suo valore di verità: circostanza a volte banale ma da sottolineare perché gioca un ruolo fondamentale nell'istituzione di credenze estranee al sapere sinora condiviso e alle agenzie tradizionali di verifica. Sino a erodere gli stessi criteri veritativi in vigore (Stefano Bartezzaghi, Banalitá, luoghi comuni, semiotica, social network, Milano-Firenze, Bompiani-Giunti, 2019, p. 68).
\end{abstract}

L'accezione di 'incolto' nasce dal fatto che l'istruzione propone, attraverso una serie di prove, il modello della Terra sferica, ormai accolto come verità scientificamente provata e dunque solo chi abbia frequentato poco e male la scuola possa mettere in dubbio un fatto ampiamente e variamente dimostrabile come la sfericità della Terra. Di conseguenza si sviluppa l'accezione di 'idiota', ovvero 'persona poco intelligente', incapace di comprendere tutte le tesi scientifiche che sostengono la rotondità della Terra (dalle leggi sulla gravità, alla rifrazione della luce ecc.), e che si basa solo su ciò che l'occhio umano, nella sua capacità sensibile, riesce a vedere ovvero l'orizzonte piatto. A tal proposito sono significative le parole di un giornalista del "Corriere della Sera", quando spiega le affermazioni di Beppe Grillo comparse sul suo blog:

Non è un caso che Beppe Grillo per difendersi dagli attacchi dei no-vax, dopo il suo cambio di posizione sul tema, li abbia etichettati come "terrapiattisti", sinonimo dell'ottusità di chi non riconosce la 
superiorità del metodo introdotto proprio da Galilei (Massimo Sideri, Il patto anti fake news tra scienza e umanesimo, in "Corriere della Sera", XVIII, I44, p. 24).

L'accezione di 'idiota', soprattutto con funzione di insulto, è quella più popolare:

Eccolo con le scie chimiche, aggiunga subito anche terrapiattista che per screditare ultimamente sta andando molto di moda ( tweet di @Cristia59109404 (Cris P.I.), 20/5/2019).

uhm quindi io sarei un coglione terrapiattista...grazie... forse mentre prendevo il dottorato in strumentazione elettronica di misura devo essermi distratto ( tweet di @Gondori8I40506 (Gondor), I $9 / 5 / 2019)$.

Quindi ci mettiamo dei no vax, qualche terrapiattista al comando, perché gli idioti li servono sempre, fosse mai che casalercio si scopre per quello che è... ( tweet di @lauriargro74 (lauria), 13/5/2019).

Anche in inglese la parola flat-earther ha assunto questa accezione tant'e che nell' Urban Dictionary (Dizionario gergale angloamericano in rete le cui schede sono compilate dagli utenti), tra le varie definizioni proposte, ricorrono quelle di 'idiot' e 'very stupid' o anche 'who is mentally retarded'. L'altra accezione, quella di 'ingenuo', ha una sfumatura meno negativa e la si può cogliere nelle parole di Beppe Grillo nel post dal titolo "Amate e coccolate il terrapiattista che è in voi":

Insomma il bambino che c'è in noi è il primo ad essere terrapiattista! E' [sic] dura ammetterlo ma è così, sono tempi difficili e densi di buie prese di coscienza. [...] Non si faceva prima a coccolare il fanciullino terrapiattista che c'è in noi, invece di negarne l'esistenza? Ebbene coccolatelo, viziatelo, perché è meglio credere a delle innocue stupidaggini che prendersela con gli altri, non ci credete? ( post sul blog beppegrillo.it, 9/4/2019).

Terrapiattista compare anche con altre sfumature di significato meno frequenti come persona con manie di persecuzione' (accezione che si è sviluppata a partire dalle ipotesi complottiste) o, al contrario 'anticonformista', in quanto sfiderebbe l'idea conformistica della Terra tonda nonché la dittatura della scienza. Quest'ultima accezione viene più comunemente usata dai terrapiattisti stessi.

\# Terrapiattisti. / La stampa non fa che parlarne. / Perché? / Noterete che le loro idiozie vengono associate alle critiche ad immigrazione e vaccinazioni invasive. / Dunque? / Il messaggio è: criticare il sistema è ignoranza da «terrapiattisti». / Come serpe, la dittatura s'insinua ovunque ( tweet di @intuslegens (il Sofista), 13/5/2019).

"Un terrapiattista è una persona che per motivi intellettuali riesce a comprendere cose che la gente comune non è capace di capire. La gente comune è ingabbiata da dottrine che vengono instillate sin dalle elementari e poi con gli studi, la televisione e i media" \# terrapiattisti ( tweet di @e_terranova (Elvira Terranova), I2/5/2019).

In funzione ironica e derisoria è stato coniato anche il termine terrapiattaro che sostituisce il suffisso -ista con -aro: al plurale (terrapiattari) registra su Google ben 3.Ioo occorrenze il 17/5/2019 (532 per terrapiattaro).

Adoro il lavoro che fate in questo blog perché è nutrimento per le menti curiose e aperte, ma $\mathrm{i}$ terrapiattari non sono ne curiosi né aperti, hanno già le loro certezze e li fanno stare bene ( commento di Anonimo sul blog flatearthdelusion.blogspot.com, 7/I2/2017).

La parola terrapiattista, infine, ha fornito spunto per la creazione di una serie di altri composti che, ad 
oggi, possono essere considerati solo occasionalismi visto che non registrano una frequenza d'uso rilevante o tale da poterceli far considerare parole nuove. I composti a partire da Terra sono tanti: $\mathrm{i}$ sostenitori della Terra sferica possono essere chiamati terratondisti, terrasferisti oterraglobisti (su internet prevale il primo composto), ma si hanno anche, con intenti più spiccatamente ironici, i sostenitori della terra cubica (terracubisti con il loro blog satirico the Cubic Earth Society) e quadrata (terraquadratisti), concava e cava (terraconcavisti e terracavisti).

Se fossi terrapiattista avrei solo questa espressione...ma sono un attore e la posso cambiare.Buona serata terratondisti!!! ( tweet di @GassmannGassmann (Alessandro Gassmann), I2/5/2019).

Io comunque sono un terracavista...molto più plausibile.. come nei racconti di Jules Verne, e poi lo sanno tutti che anche la luna è cava, hanno fatto gli esperimenti e confermano questo... ( tweet di @F_enrico88, 17/5/2019).

Sempre in maniera ironica e giocosa, non mancano i composti formati a partire da -piattista per indicare il 'sostenitore che qualcosa sia piatto':

Vorrei entrare nel club dei panciapiattisti. Ma sarebbe più inverosimile dei terrapiattisti ( @andreavianel (Andrea Vianello), I2/5/2019).

E comunque i peggiori sono i panciapiattisti, quelli che credono nelle pance piatte durante il Natale ( @opificioprugna (Prugna), 20/12/2019).

La parola terrapiattismo invece non fornisce nessuno spunto per la creazione di parole nuove: infatti terratondisti e terrasferisti registrano su Google rispettivamente 3.890 e 2.350 occ. mentre terratondismo e terrasferismo solo 50 e 63 (dati del I6/5/2019). Situazione simile per tutti gli altri composti. Infine gli sviluppi semantici che ha registrato la parola terrapiattista ci fanno considerare quest'ultimo termine molto più vitale rispetto a terrapiattismo, che nasce probabilmente come conseguenza della diffusione di terrapiattista e viene spesso sostituito da Terra piatta o movimento della Terra piatta.

\section{Cita come:}

Miriam Di Carlo, //terrapiattismo e i suoi sostenitori, "Italiano digitale", 2019, IX, 2019/2 (aprilegiugno)

DOI: $10.35948 / 2532-9006 / 2020.3149$ 